\title{
GRUPO OPERATIVO COM ESTUDANTES DE ENFERMAGEM: VIVÊNCIA EM UMA LIGA ACADÊMICA DE ONCOLOGIA
}

\author{
OPERATIVE GROUP WITH NURSING STUDENTS: \\ EXPERIENCE IN AN ONCOLOGY ACADEMIC LEAGUE
}

\section{GRUPO OPERATIVO CON ESTUDIANTES DE ENFERMERÍA: EXPERIENCIA EN UNA LIGA ACADÉMICA DE ONCOLOGÍA}

\author{
Lucas Rossato $^{1}$ \\ Marislei Sanches Panobianco ${ }^{2}$ \\ Fabio Scorsolini-Comin ${ }^{3}$
}

\begin{abstract}
Como citar este artigo: Rossato L, Panobianco MS, Scorsolini-Comin F. Grupo operativo com estudantes de enfermagem: vivência em uma Liga Acadêmica de Oncologia. Rev baiana enferm. 2020;34:e34690.

Objetivo: descrever uma atividade grupal desenvolvida em uma Liga Acadêmica de Prevenção ao Câncer. Método: relato de experiência em um grupo operativo realizado no primeiro semestre de 2019, em uma universidade pública do interior de São Paulo, com 27 graduandos do curso de Enfermagem com idades entre 17 e 25 anos, cursando entre o primeiro e o sétimo períodos letivos. Resultados: nas discussões grupais emergiram quatro temas, evidenciando aspectos do contexto de adoecimento por câncer, como estigmas, medos, anseios, fantasias, vivências do câncer como uma doença agressiva e limitante para o acometido e sua família, além de recursos de enfrentamento, como a religiosidade/espiritualidade. Conclusão: a atividade grupal desenvolvida em uma Liga Acadêmica de Prevenção ao Câncer evidenciou a importância de a oncologia ser trabalhada na formação de Enfermagem, capacitando os estudantes para lidarem com situações de intervenção e prepararem-se emocionalmente para os desafios que podem emergir nesse campo de atuação futura.
\end{abstract}

Descritores: Educação em Enfermagem. Educação em Saúde. Grupos Focais. Oncologia. Psico-Oncologia.

Objective: to describe a group activity developed in an Academic League for Cancer Prevention. Method: an experience report on an operative group held in the first half of 2019, at a public university in the countryside of São Paulo, with 27 students from the Nursing course aged between 17 and 25 years, attending between the first and seventh school terms. Results: in group discussions, four themes emerged, highlighting aspects of the context of illness from cancer, such as stigmas, fears, desires, fantasies, experiences of cancer as an aggressive and limiting disease for the affected and their families, in addition to coping resources, such as religion/spirituality. Conclusion: the group activity developed in an Academic League for Cancer Prevention revealed the importance of addressing oncology in Nursing graduation, enabling students to deal with situations of intervention and prepare themselves emotionally for the challenges that may emerge in this field in the future.

Descriptors: Education, Nursing. Health Education. Focus Groups. Medical Oncology. Psycho-Oncology.

\footnotetext{
Psicólogo. Mestre em Psicologia. Universidade de São Paulo. Ribeirão Preto, São Paulo, Brasil. rossatousp@usp.br. https://orcid.org/0000-0003-3350-0688. Enfermeira. Doutora em Enfermagem em Saúde Pública. Livre-Docente da Universidade de São Paulo. Ribeirão Preto, São Paulo, Brasil. https://orcid.org/00000003-2619-8740.
}

3 Psicólogo. Doutor em Psicologia. Professor da Universidade de São Paulo. Ribeirão Preto, São Paulo, Brasil. https://orcid.org/0000-000 I-628I-337l . 
Objetivo: describir una actividad en grupo desarrollada en una Liga Académica para Prevención al Cáncer. Método: relato de experiencia de un grupo operativo celebrado en el primer semestre de 2019, en una universidad pública en el interior de São Paulo, con 27 estudiantes del curso de Enfermería con edades comprendidas entre los $17 y$ los 25 años, cursando entre el primer y el séptimo períodos lectivos. Resultados: en las discusiones en grupo, surgieron cuatro temas, destacando aspectos del contexto de enfermedad causada por el cáncer, tales como estigmas, miedos, deseos, fantasias, experiencias de cáncer como una enfermedad agresiva y limitante para los afectados y sus familias, además de recursos de afrontamiento, tales como la religión/espiritualidad. Conclusión: las actividades en grupo desarrolladas en una Liga Académica para Prevención al Cáncer mostró la importancia de trabajarse la oncología en la formación de Enfermería, permitiendo a los estudiantes lidiar con situaciones de intervención y prepararse emocionalmente para los desafíos que puedan surgir en este campo en el futuro.

Descriptores: Educación en Enfermería. Educación en Salud. Grupos Focales. Oncología Médica. Psicooncología.

\section{Introdução}

$\mathrm{Na}$ formação profissional nas áreas da saúde tem ocorrido uma preocupação com a transmissão de conteúdos e a inserção em atividades práticas que possibilitem a compreensão dos sujeitos em sua integralidade, por meio de ações de ensino, pesquisa e extensão. Entre as estratégias adotadas por acadêmicos para participação mais ativa no processo formativo e de possibilidade de praticar atividades de extensão universitária que os coloquem em contato direto com temáticas de interesse, complementares aos conteúdos regulares, estão as Ligas Acadêmicas ${ }^{(1)}$. Estas são conceituadas como grupos mantidos e organizados por estudantes de graduação, que possuem estatuto próprio e gozam de autonomia para a realização de atividades e eventos, respeitando as normativas das unidades educacionais às quais estão submetidas, como departamentos, unidades de ensino e a própria universidade. Nesses espaços, os estudantes podem organizar ações que visem à ampliação de conhecimentos em determinadas áreas, aumentando o repertório oferecido nas grades curriculares. Além disso, permitem o engajamento dos alunos em ações de formação e também de contato com a comunidade, notadamente nos cursos da área de saúde, entre eles a Enfermagem, como priorizado no presente relato de experiência.

Assim, as Ligas Acadêmicas integram ações dos três aspectos da formação universitária: o ensino, a pesquisa e a extensão. Esta última é de interesse deste relato de experiência. O enfoque dado aqui será referente à atividade desenvolvida junto à Liga de Prevenção e Combate ao
Câncer (LPCC) que funciona, desde o ano de 1998, em uma instituição pública localizada no interior do estado de São Paulo.

O câncer tem sido enfatizado nas últimas décadas como um problema de saúde pública, nacional e internacionalmente, de grande relevância epidemiológica no que se refere à incidência e morbimortalidade. É importante que seja considerado enquanto temática na formação em Enfermagem, uma vez que essa profissão assume grandes responsabilidades frente a esses pacientes e seus familiares, na assistência, na avaliação diagnóstica, no tratamento e na reabilitação ${ }^{(2)}$. Na atualidade, o adoecimento por câncer tem se tornado um evento comum e universal, descrito pela literatura científica como um problema que atinge pessoas em diferentes faixas etárias e em diferentes localidades do mundo ${ }^{(3-6)}$. No Brasil, a partir da segunda metade do século XX, intensificaram-se as preocupações com relação a esse adoecimento. Tal processo o transformou em uma presença marcante na vida cotidiana e em uma vigorosa preocupação social ${ }^{(7)}$.

A assistência às pessoas com câncer tem avançado ao longo dos anos, devido aos novos métodos de detecção precoce, rastreamento dos agentes cancerígenos e tipos de neoplasias, entre outros aspectos ${ }^{(2)}$. Os avanços alcançados no desenvolvimento de novas tecnologias, medicamentos e procedimentos proporcionaram redução significativa no número de mortes e melhoria nos cuidados prestados ${ }^{(8)}$. 
Embora as taxas de cura tenham aumentado nas últimas décadas, o número de pessoas que vão a óbito devido a esta doença ou a complicações decorrentes dela ainda é alarmante. Segundo dados do Instituto Nacional de Câncer (INCA), só no ano de 2015, 107.470 pessoas do sexo masculino e 90.228 pessoas do sexo feminino foram a óbito acometidas por diversos tipos de neoplasias ${ }^{(9)}$. Estes dados evidenciam a problemática que esse tipo de adoecimento assume em contexto nacional, requerendo atenção dos órgãos de saúde.

Em nível internacional, cerca de uma em cada seis mortes é devido ao câncer. Este tipo de adoecimento constitui-se como a segunda principal causa de morte no mundo. Somente em 2018, foi responsável por cerca de 9,6 milhões de óbitos. É importante salientar que, dentre o número de óbitos registrados, aproximadamente 70\% ocorreram em países de baixa e média renda ${ }^{(10)}$, o que demonstra possíveis desigualdades de acesso a recursos adequados para o tratamento da doença.

A problemática que o câncer assume na atualidade requer o constante desenvolvimento $\mathrm{e}$ aprimoramento de políticas públicas para prevenção, bem como de ações ativas e resolutivas para o tratamento e acompanhamento dos sujeitos adoecidos, compreendendo-os como seres bio-psico-socioespirituais ${ }^{(11)}$. Neste sentido, é importante ressaltar que, para além dos aspectos biológicos, o processo de adoecimento por câncer é fortemente marcado por aspectos psicossociais $^{(12)}$ socioeconômicos ${ }^{(13-15)}$ e culturais ${ }^{(16)}$ que merecem ser considerados.

Esses aspectos, em muitas situações interferirão no surgimento de estigmas, fantasias, medos e receios em relação ao adoecer, na aceitação ou recusa de tratamento, bem como no acesso às condições dignas de tratamento. Dessa forma, impactará nos índices de sobrevivência e na qualidade de vida das pessoas adoecidas, de familiares, cuidadores e da equipe de saúde que acompanhará. Assim, na graduação em Enfermagem, torna-se importante mostrar aos estudantes a complexidade do cuidado para com pacientes e familiares em situação de adoecimento por câncer, considerando que a oncologia constitui-se em um campo de atuação que tem inserido um número significativo desses profissionais.

Caberia, desse modo, às instituições de ensino repensarem suas estratégias e prioridades para a formação acadêmica (neste caso, não somente os da Enfermagem), especialmente na área da oncologia. Esses profissionais prestarão assistência a uma população em constante crescimento, com estimativa de vida cada vez maior e que procura os serviços de saúde para atendimento de necessidades que emergem em um panorama no qual a incidência do câncer aumenta a cada ano ${ }^{(1)}$. Na promoção do cuidado prestado pela equipe de enfermagem, uma das complexidades das ações a serem desenvolvidas está no fato de os profissionais terem de atentar para aspectos biopsicossociais que permeiam as vivências dos sujeitos adoecidos e das pessoas que os acompanham.

Esses aspectos são de observação essencial no processo de tratamento, pois a subjetividade da pessoa adoecida e de seus familiares, somada aos aspectos culturais, econômicos e religiosos/espirituais estarão presentes desde o diagnóstico até o final do tratamento. Nesse sentido, os profissionais devem estar atentos a esses aspectos, uma vez que, formar relações positivas com pacientes com câncer e suas famílias, bem como abordar os problemas emocionais dos pacientes em relação às suas doenças ${ }^{(17)}$, considerar suas opiniões, crenças pessoais e religiosas/espirituais, é essencial para a satisfação dos adoecidos e de seus familiares/cuidadores em relação ao cuidado recebido.

Concomitante ao cuidado prestado aos pacientes e a seus familiares/cuidadores, os profissionais devem estar atentos para o cuidado de si. Não podem esquecer-se de que terão de lidar com temas que são de vivência e elaboração dif́ceis, o que torna importante o desenvolvimento dessa percepção desde a graduação.

O destaque para a atividade grupal na graduação do curso de Enfermagem visa discutir em que medida essa metodologia ativa pode 
ser empregada nesse cenário de interface entre extensão e ensino, possibilitando maior engajamento do aluno e oportunizando expressões que fazem parte de sua formação profissional e construção de sua identidade futura como enfermeiro(a).

Assim, o objetivo deste relato de experiência é descrever uma atividade grupal desenvolvida em uma Liga Acadêmica de Prevenção e Combate ao Câncer.

\section{Método}

Trata-se de um relato de experiência realizado no primeiro semestre de 2019 com estudantes de um curso de graduação em Enfermagem de uma instituição de ensino superior do interior de São Paulo, convidados a descrever suas experiências pessoais e acadêmicas com a oncologia. Participaram 27 universitários, sendo 24 do gênero feminino e 3 do gênero masculino, com idades entre 17 e 25 anos e cursando entre o $1^{\circ}$ e o $7^{\circ}$ períodos letivos. Também estavam presentes uma professora do curso de Enfermagem e o psicólogo que coordenou as atividades grupais.

A atividade grupal teve como objetivo fomentar a participação dos graduandos, bem como trazer elementos identitários que pudessem ser úteis tanto nos estudos como na preparação para futuras intervenções da enfermagem no campo da oncologia. Assim, foi desenvolvido um grupo operativo de ensino-aprendizagem, por meio da técnica de grupos de reflexão, embasados no modelo de Pichon-Rivière ${ }^{(18)}$, em um único encontro.

Os grupos operativos constituem-se como um dos diversos instrumentos disponíveis para intervenções grupais. O desenvolvimento de grupos operativos parte do pressuposto da organização de um conjunto de pessoas com objetivo comum, que procuram abordar uma temática e trabalhar em equipe. Neste processo, a técnica consiste em analisar os aspectos implícitos ou latentes e os aspectos manifestos pelos participantes, tornando consciente o inconsciente. A atividade grupal parte da premissa básica de transmudar situações estereotipadas e dilemáticas em situações dialéticas e que levem ao aprendizado ${ }^{(18)}$. A aprendizagem deve ser considerada um processo de avanços e recuos em um constante movimento dialético. Enquanto se aprende, abandonam-se formas de ver o mundo ou uma realidade, passando a ter um outro olhar sobre o que está sendo abordado. Essa modalidade grupal está estruturada em um modelo de atuação centrado em uma tarefa que é definida no início das atividades e orientará o trabalho grupal.

O encontro ocorreu em uma sala de aula da universidade, sendo organizado um círculo no qual os participantes pudessem se ver e ouvir. O tempo de duração foi de uma hora e cinco minutos, sendo a tarefa grupal acordada entre os participantes, conforme a solicitação da Liga, mas direcionada de acordo com os conteúdos que emergiram do campo grupal, sendo: vivências pessoais e acadêmicas em que tiveram contato com pessoas com câncer e sobre os conhecimentos que tinham sobre a temática antes e após entrarem no curso de Enfermagem.

O conteúdo foi analisado por meio da escuta do material. A organização dos dados foi realizada mediante o levantamento dos temas emergentes do grupo e foram apresentados na ordem sequencial em que apareceram. Os conteúdos manifestados foram analisados e discutidos à luz da literatura científica sobre o adoecimento por câncer.

\section{Resultados e Discussão}

Com base nas discussões, observou-se, como conteúdo dos diálogos, quatro temas emergentes que serão apresentados a seguir. Para iniciar as atividades, foi proposto aos estudantes que discorressem sobre o que lhes vinha à cabeça quando ouviam a palavra câncer. O objetivo era entrar na tarefa grupal por meio de uma temática que não evocasse logo no início os aspectos pessoais relacionados às vivências da doença. Sabe-se que essa temática não é fácil de ser abordada, devido às representações simbólicas que, muitas vezes, se encontram associadas à morte.

\section{Primeiro tema emergente: Percepções pessoais sobre a palaura câncer}

Ao discorrerem sobre o que pensavam quando ouviam a palavra câncer, os estudantes descreveram aspectos relacionados ao tratamento da 
doença, como, por exemplo, a quimioterapia, os aspectos biológicos do adoecer e o estigma atrelado à palavra câncer, como um sinônimo de morte iminente, uma vez que a pessoa ou morre pelo câncer, ou por outro processo de adoecimento que advém do câncer. Em relação ao fato de discorrerem sobre os aspectos biológicos da doença, é plausível que os estudantes abordem este assunto, uma vez que, por estarem em um curso da área da saúde, são constantemente interpelados a compreender os sentidos biológicos do adoecer, em um paradigma que muitas vezes ainda é biomédico.

Em relação às percepções pessoais sobre o câncer, há de ser considerado que as representações que as pessoas desenvolvem sobre uma temática são influenciadas diretamente por aspectos históricos, sociais, culturais, políticos e econômicos que moldarão crenças, valores, comportamentos, percepções, emoções e a subjetividade de modo geral ${ }^{(19)}$. Desse modo, é possível compreender que as percepções construídas pelos estudantes participantes do grupo sobre a palavra câncer estivessem relacionadas à concepção do câncer como uma doença complexa e demarcada por significados negativos que lhe são socialmente atribuídos.

\section{Segundo tema emergente: Percepçôes e conhecimentos sobre o câncer antes e depois da entrada na universidade}

Neste tema, os estudantes descreveram que não tinham muito conhecimento sobre a doença antes de entrar no curso de enfermagem. O que sabiam referia-se ao conhecimento popular (e muitas vezes estigmatizado) de que o câncer é uma doença sem cura, que acomete a pessoa e a leva a óbito em pouco tempo. Este aspecto relatado pelo grupo em relação ao adoecimento por câncer está atrelado ao imaginário social do câncer enquanto doença terminal, sem perspectiva de cura e carregado de sofrimento, interligado ao que abordaram no primeiro tema emergente.

É importante salientar que essas percepções sobre o adoecimento não estão de todo equivocadas. O câncer constitui-se realmente como um conjunto de doenças complexas, que requerem atenção e cuidado, com procedimentos invasivos, que causam dor, afetando física e psicologicamente as pessoas e seus familiares. É um importante problema de saúde pública não só em países em desenvolvimento como também nos desenvolvidos ${ }^{(20)}$. Contudo, não se resume a isso, pois os avanços nos procedimentos adotados para tratamento, somados às novas formas de cuidado ofertadas possibilitam, na atualidade, novas perspectivas em relação à doença.

A formação em Enfermagem deve, assim, estar atenta para a dinâmica de atendimento prestado aos pacientes oncológicos e seus familiares. Atividades como as desenvolvidas pela Liga de Prevenção e Combate ao Câncer tornam-se ações complementares fundamentais para enriquecer o repertório da formação universitária, preparando o estudante, de certa forma, para contextos e situações que ele possa vir a enfrentar na enfermagem oncológica.

\section{Terceiro tema emergente: Estigma em relação ao câncer}

O estigma em relação ao câncer foi outro tema que emergiu nos debates. Os participantes referiram que o estigma em relação ao câncer levava as pessoas a não falar sobre o assunto. Nas situações em que a pessoa já está adoecida, ela pode não revelar o adoecimento, por temer que o ato de falar sobre a doença desperte uma série de sentimentos e comportamentos nas outras pessoas. Há também as situações em que não se discorre sobre o câncer, pela fantasia de que o ato de falar sobre essa doença poderia atraí-la. Desse modo, percebe-se a dimensão dos significados socialmente atribuídos ao câncer e o impacto disso na vida das pessoas, particularmente dos estudantes de Enfermagem.

Sabe-se que, culturalmente, esse aspecto está arraigado na sociedade e influencia e compromete as relações dos sujeitos adoecidos com familiares e com o grupo social a que pertence, haja vista que ocasiona dificuldade de falar sobre a doença de forma aberta e franca ${ }^{(21)}$. O câncer tem sido apresentado pela literatura 
científica como um processo atrelado às percepções sociais $^{(19)}$, da pessoa adoecida, dos familiares e até mesmo dos profissionais de saúde em relação à doença.

Apesar de toda a evolução tecnológica e das formas de tratamento, o estigma do câncer imprime uma marca na cultura, repleta de representações negativas que parecem não se dissolver ${ }^{(19)}$. Este aspecto precisa ser considerado na atuação dos profissionais que prestam cuidado, uma vez que interfere na prevenção, no momento do diagnóstico, na adesão ao tratamento e durante o tratamento ${ }^{(22)}$, despertando sentimentos diversos, como tristeza, indignação, angústia, medo da morte, entre outros.

\section{Quarto tema emergente: Apoio à pessoa adoecida por câncer}

Sobre o apoio que a pessoa recebe, o prestado pela família foi descrito como essencial. Muitas vezes, a pessoa que recebe a notícia fica desamparada e a família, embora sofrendo junto, acaba dando suporte. Transmitir segurança para a pessoa é essencial, mesmo sabendo que pode não dar certo. O apoio ofertado vai desde questões práticas, que a pessoa adoecida precisa para desempenhar certas atividades, como o apoio emocional, que vai desde o acolhimento até o suporte e encorajamento. A rede de apoio deve conceder, assim, duas instâncias de auxílio aos seus pacientes: a instrumental e a emocional ${ }^{(23)}$. Em relação ao suporte social, foi comentado sobre a importância do apoio religioso/espiritual, respeitando-se, no entanto, a orientação religiosa/espiritual de cada sujeito ou ainda o fato de que alguns podiam não seguir nenhuma orientação.

Sabe-se que a influência do câncer na dinâmica familiar é marcada por temores não só do adoecido como também das pessoas que o acompanham. Considerando-se que as neoplasias têm como características a possibilidade de recidiva e a necessidade de intervenção, é natural que esse fenômeno altere o contexto familiar, pois expõe as pessoas a maior vulnerabilidade, perdas de saúde e bem-estar, perdas financeiras e de equilíbrio físico, mental e emocional ${ }^{(20)}$.
O impacto do adoecimento por câncer deve ser observado, considerando as repercussões na vida do sujeito acometido pela doença e na dinâmica familiar, já que todos estarão envolvidos no processo. Este aspecto tem relevância significativa na formação em Enfermagem, pois é importante alertar os graduandos para a necessidade de atentarem não somente às necessidades físicas dos pacientes e familiares, mas também aos aspectos subjetivos que permeiam o cuidado. A Enfermagem, junto com os demais membros da equipe profissional, possui um papel essencial para evitar o abandono do tratamento.

Esses temas emergentes possibilitam o reconhecimento da dimensão mobilizadora do grupo com esses alunos. Ainda que aspectos ligados aos conhecimentos acadêmicos acerca dessa temática sejam recuperados e discutidos, elementos emocionais centralizados nos próprios alunos puderam ser trazidos à baila e dimensionados em um espaço de formação construído por alunos e coordenadores da atividade. Assim, recomenda-se o grupo não apenas como convite inicial para abordagem do câncer, mas também como espaço perene de reflexão sobre a maneira de cada aluno deparar-se com a oncologia ao longo do seu percurso estudantil na graduação em Enfermagem.

Como limitador deste estudo, o fato de ser uma única atividade grupal insere-o em um contexto específico, com suas particularidades, que devem ser consideradas. Além do mais, vários estudantes presentes eram calouros e os significados atribuídos ao câncer devem ser vistos com ressalvas, uma vez que ainda não haviam tido contato prático e profissional com a área da oncologia.

\section{Conclusão}

A experiência do grupo com os estudantes de Enfermagem evidenciou aspectos que fazem parte do contexto de adoecimento por câncer, como estigmas, medos, anseios, fantasias, vivências marcadas por sentimentos do câncer como uma doença agressiva e que impõe limitações para a pessoa adoecida com repercussão no ambiente familiar. Tais aspectos demonstram a importância de a temática ser trabalhada nos 
contextos de formação de Enfermagem, a fim de que os sujeitos adquiram repertório teórico e técnico para lidar nas situações de intervenção na oncologia, bem como sejam preparados psicologicamente para os desafios que podem enfrentar nesse campo de trabalho.

O grupo mostrou-se como um espaço em que os estudantes tiveram a possibilidade de apresentar suas vivências pessoais e acadêmicas sobre o câncer e foi marcado por ser um momento de tensões e com períodos de silêncio que denotavam a dificuldade em abordar a temática. Sabe-se que, durante a formação, algumas temáticas abordadas nas disciplinas ou as experiências vivenciadas nas práticas de estágio, pesquisa e extensão podem ser intensas para os estudantes e nem sempre são bem elaboradas. Por isso, disporem de um espaço para refletir e verbalizar sobre essas vivências é pertinente, para que sejam compreendidas ansiedades, angústias e sentimentos vividos por eles e que podem interferir na sua formação e também em suas condições de saúde mental. O levantamento dos temas emergentes demonstrou o movimento percorrido durante a tarefa grupal e tornou possível a apresentação das temáticas mais recorrentes.

\section{Fonte de Financiamento:}

Coordenação de Aperfeiçoamento de Pessoal de Nível Superior (CAPES).

\section{Colaborações:}

1 - concepção, projeto, análise e interpretação dos dados: Lucas Rossato, Marislei Sanches Panobianco e Fabio Scorsolini-Comin;

2 - redação do artigo e revisão crítica relevante do conteúdo intelectual: Lucas Rossato, Marislei Sanches Panobianco e Fabio Scorsolini-Comin;

3 - aprovação final da versão a ser publicada: Lucas Rossato, Marislei Sanches Panobianco e Fabio Scorsolini-Comin.

\section{Referências}

1. Panobianco MS, Borges ML, Caetano EA, Sampaio BAL, Magalhães PAP, Moraes DC. A contribuição de uma Liga Acadêmica no ensino de graduação em Enfermagem. Rev Rene [Internet]. 2013 [cited 2019 Nov 25];14(1):169-78. Available from: http://periodicos.ufc.br/rene/article/ view/3351/2589

2. Luz KR, Vargas MAO, Barlem ELD, Schmitt PH, Ramos FRS, Meirelles BHS. Coping strategies for oncology nurses in high complexity. Rev Bras Enferm. 2016 Jan/Feb;69(1):59-63. DOI: http:// dx.doi.org/10.1590/0034-7167.2016690109i

3. Li Y, Qiao Y, Luan X, Li S, Wang K. Family resilience and psychological well-being among Chinese breast cancer survivors and their caregivers. Eur J Cancer Care. 2019 Mar;28(2):e12984. DOI: 10.1111/ ecc. 12984

4. Martins HTG, Balmant NV, de Paula Silva N, Santos MO, Reis RS, de Camargo B. Who cares for adolescents and young adults with cancer in Brazil? J Pediatr. 2018 Jul-Aug;94(4):440-5. DOI: 10.1016/j.jped.2017.07.008

5. Muzatti B, Gipponi K, Flaiban C, Cormio C, Carnaghi C, Tralongo $\mathrm{P}$, et al. The impact of cancer: An Italian descriptive study involving 500 long-term cancer survivors. Eur J Cancer Care. 2019 May;28(3):e13007. DOI: 10.1111/ecc.13007

6. Makhoul Khoury S, Ben-Zur H, Ben-Arush M. Mastery and social support moderate the effects of educational level on adjustment of Arab mothers of children diagnosed with cancer. Eur J Cancer Care. 2018 Nov;27(6):e12906. DOI: 10.1111/ ecc.12906

7. Araújo Neto LA, Teixeira LA. De doença da civilização a problema de saúde pública: Câncer, sociedade e medicina brasileira no século XX. Bol Mus Para Emílio Goeldi. Ciênc Hum. 2017;12(1):173-88. DOI: http://dx.doi.org/10.1590 /1981.81222017000100010

8. Heymach J, Krilov L, Alberg A, Baxter N, Chang SM, Corcoran RB, et al. Clinical cancer advances 2018: Annual report on progress against cancer from the American Society of Clinical Oncology. J Clin Oncol. 2018 Apr;36(10):1020-44. DOI: $10.1200 /$ JCO.2017.77.0446

9. Brasil. Ministério da Saúde. Instituto Nacional do Câncer José Alencar Gomes da Silva. Câncer [Internet]. Rio de Janeiro; 2018 [cited 2019 Nov 25]. Available from: https://www.inca.gov.br/

10. World Health Organization. Cancer [Internet]. Genève; 2019 [cited 2019 Nov 25]. Available from: https://www.who.int/news-room/fact-sheets/ detail/cancer 
11. World Health Organization. Division of mental health and prevention of substance abuse. WHOQOL and spirituality, religiousness and personal beliefs (SRPB) [Internet]. Genève; 1998 [cited 2019 Nov 25]. Available from: https://apps. who.int/iris/handle/10665/70897

12. Johansen C, Rosberger Z. Psychosocial aspects of cancer need integration into the treatment trajectory-but how? Cur Oncol. 2012 Aug;19(4):193-4. DOI: 10.3747/co.19.1160

13. Cazap E, Magrath I, Kingham TP, Elzawawy A. Structural barriers to diagnosis and treatment of cancer in low- and middle-income countries: The urgent need for scaling up. J Clin Oncol. 2015 Jan;34(1):14-9. DOI: 10.1200/JCO.2015.61.9189

14. Rodrigues JD, Cruz MS, Paixao AN. Uma análise da prevenção do câncer de mama no Brasil. Ciênc Saúde Coletiva. 2015;20(10):3163-76. DOI: http://dx.doi.org/10.1590/1413-812320152010.20822014

15. Smith DK, Castellanos EH, Murphy BA. Financial and socio-economic factors influencing pre- and post-cancer therapy oral care. Support Care Cancer. 2018 Jul;26(7):2143-8. DOI: 10.1007/ s00520-017-4033-9

16. Palacios-Espinosa X, Zani B. La stigmatisation de la maladie physique: Le cas du câncer. Psycho-Oncol [Internet]. 2012 Dec [cited 2019 Nov 25];6(4):189200. Available from: https://link.springer.com/ article/10.1007/s11839-012-0391-1

17. Kapucu S, Bulut HD. Nursing students' perspectives on assisting cancer patients. Asia Pac J Oncol Nurs. 2018 Jan-Mar;5(1):99-106. DOI: 10.4103/apjon. apjon_44_17
18. Pichon-Rivière E. O processo grupal. São Paulo: Martins Fontes; 2009.

19. Barbosa LNF, Francisco AL. A subjetividade do câncer na cultura: implicações na clínica contemporânea. Rev SBPH [Internet]. 2007 jun [cited 2019 Nov 25];10(1):9-24. Available from: http://pepsic.bvsalud.org/scielo.php?script=sci_ arttext\&pid=S1516-08582007000100003

20. Sales CA, Matos PCB, Mendonça DPR, Marcon SS. Cuidar de um familiar com câncer: o impacto no cotidiano de vida do cuidador. Rev Eletrôn Enferm. 2010;12(4):616-21. DOI: 10.5216/ree.v12i4.12160

21. Mattos K, Blomer TH, Campos ACBF, Silvério MR. Estratégias de enfrentamento do câncer adotadas por familiares de indivíduos em tratamento oncológico. Rev Psicol Saúde. 2016 jun;8(1):1-6. DOI: http://dx.doi.org/10.20435/2177093X2016101

22. Costa JM, Finco GM, Souza RLG, Medeiros WCM, Melo MCM. Repercussões biopsicossociais do diagnóstico de câncer colorretal para pacientes oncológicos. Rev SBPH [Internet]. 2016 [cited 2019 Nov 25];19(2):5-23. Available from: http:// pepsic.bvsalud.org/scielo.php?script $=$ sci arttext\&pid=\$1516-08582016000200002

23. Ferreira PC, Waktuchi J, Baldissera VDA, Sales CA. Sentimentos existenciais expressos por usuários da casa de apoio para pessoas com câncer. Esc Anna Nery. 2015;19(1):66-72. DOI: http://dx.doi. org/10.5935/1414-8145.20150009

Recebido: 25 de novembro de 2019

Aprovado: 10 de dezembro de 2019

Publicado: 2 de abril de 2020

A Revista Baiana de Enfermagem utiliza a Licença Creative Commons - Atribuição-NãoComercial 4.0 Internacional. https://creativecommons.org/licenses/by-nc/4.0/

Este artigo é de acesso aberto distribuído sob os termos da Licença Creative Commons (CC BY-NC).

Esta licença permite que outros remixem, adaptem e criem a partir do seu trabalho para fins não comerciais. Embora os novos trabalhos tenham de lhe atribuir o devido crédito e não possam ser usados para fins comerciais, os usuários não têm de licenciar esses trabalhos derivados sob os mesmos termos. 